\title{
Improving double-ended transition state searches for soft-matter systems
}

\author{
K. Röder ${ }^{1}$ and D. J. Wales ${ }^{1, a)}$ \\ Department of Chemistry, University of Cambridge, Lensfield Road, CB2 1EW, Cambridge, \\ $U K$.
}

(Dated: 19 June 2020)

Transitions between different stable configurations of biomolecules are important in understanding disease mechanisms, structure-function relations, and for novel molecular-scale engineering. The corresponding pathways can be characterised efficiently using geometry optimisation schemes based on double-ended transition state searches. An interpolation is first constructed between the known states, and then refined, yielding a band that contains transition state candidates. Here we analyse an example where various interpolation schemes lead to bands with a single step transition, but the correct pathway actually proceeds via an intervening, low-energy minimum. We compare a number of different interpolation schemes for this problem.

We systematically alter the number of discrete images in the interpolations and the spring constants used in the optimisation, and test two schemes for adjusting the spring constants and image distribution, resulting in a total of 2,760 different connection attempts. Our results confirm that optimised bands are not necessarily a good description of the transition pathways in themselves, and further refinement to actually converge transition states and establish their connectivity is required. We see an improvement in the optimised bands if we employ the adjustment of spring constants with DNEB, and a smaller improvement from the image redistribution. The example we consider is representative of numerous cases we have encountered in a wide variety of molecular and condensed matter systems.

\section{INTRODUCTION}

The information necessary to understand the behaviour of molecular systems is encoded in the underlying potential energy landscape. ${ }^{1}$ This fundamental link is of particular interest in biomolecular studies, where the energy landscape picture provides insight into folding pathways and disease mechanisms. ${ }^{2-5}$ Consequently, most simulation studies target, directly or indirectly, this high-dimensional surface, and aim to explore it to predict and explain observable properties.

A straightforward approach is provided by computing the propagation of the system in time using molecular dynamics (MD). While MD simulations are perhaps the most commonly used tool in computational studies of biomolecules, it may be difficult to obtain converged results. The first challenge encountered when studying biomolecules is the large number of degrees of freedom, in common with practically any other simulation technique applied to molecular biology. Secondly, biomolecules may exhibit broken ergodicity, ${ }^{6}$ with high barriers separating structures of interest. Prohibitively large simulation times are then required for standard sampling methods, which motivates the development of techniques to treat rare events. $^{7-35}$

In MD replica exchange ${ }^{36}$ multiple copies of the system are propagated at different temperatures, allowing higher temperature replicas to overcome high energy barriers. Other approaches introduce a bias directly to the potential, to focus sampling on a specific reaction coordinate (e.g. umbrella sampling), ${ }^{37}$ to steer sampling towards unexplored regions (metadynamics), ${ }^{38}$ or to facilitate sampling of the state-tostate evolution (hyperdynamics). ${ }^{39}$ These methods are often employed alongside dimensional reduction, which can introduce artefacts into the simulations. ${ }^{10,40-43}$

\footnotetext{
a)Electronic mail: dw34@cam.ac.uk.
}

An alternative approach describes the dynamics with a master equation, ${ }^{44}$ either through the description of stationary points, or reactive pathways. ${ }^{34}$ Many methods for reactive pathway sampling exist, for example transition path sampling (TPS), ${ }^{10,11}$ transition interface sampling (TIS), ${ }^{14,15}$ forward flux sampling, ${ }^{21-23}$ and milestoning, ${ }^{16}$ to name but a few.

The direct search for stationary points, i.e. local minima and transition states, using geometry optimisation, is an alternative that is capable of yielding a full (approximate) description without the need for dimensionality reduction. ${ }^{1,33,34}$ This approach is particularly useful in tackling high energy barriers. While the location of minima is relatively straightforward, identifying and converging transition states requires more careful considerations. A discrete interpolation, such as the nudged (NEB) ${ }^{45-48}$ or doubly-nudged (DNEB) ${ }^{49,50}$ elastic band algorithm, is often used to identify potential candidates, which are subsequently refined using, for example, hybrid eigenvector-following. ${ }^{51,52}$ In this scenario, it is only necessary to optimise the images in the band sufficiently for the transition state candidates to be good starting points for the subsequent single-ended searches. ${ }^{49,53}$

In some reports, the bands are used on their own as a proxy for the true pathway, or employed to define reactive coordinates for further analysis. Here, we illustrate one example where the optimised nudged or doubly-nudged elastic bands are not necessarily sufficient to describe transitions, and converging the transition states properly along with additional sampling is required. We investigate how the choice of input parameters can produce physically sensible bands, and how adjusting the spring constants and redistributing the images in the optimisation procedure influences the outcomes. We focus on a single connection attempt, i.e. the initial interpolation, followed by an optimisation to obtain a band, and the subsequent refinement of transition state candidates. 


\section{NUDGED AND DOUBLY-NUDGED ELASTIC BANDS}

The NEB ${ }^{45-48}$ and DNEB $^{49}$ algorithms add an artificial spring potential to the true potential to connect a set of discrete images $\left[\mathbf{X}_{1}, \mathbf{X}_{2}, \ldots \mathbf{X}_{N}\right]$ between two endpoints, $\mathbf{X}_{0}$ and $\mathbf{X}_{N+1}$, where $\mathbf{X}_{i}$ is a vector containing the coordinates of image $i$. The spring potential, $V_{\mathrm{spr}}$, has the form

$$
V_{\mathrm{spr}}=\frac{1}{2} k_{\mathrm{spr}} \sum_{i=1}^{N+1}\left|\mathbf{X}_{i}-\mathbf{X}_{i-1}\right|^{2} .
$$

This approach yields an interpolation between the two endpoints, which can be optimised by minimising the energy of the entire band, approximating a minimum energy path. However, this procedure suffers from two problems: the images tend to slide downhill towards the endpoints, and in addition the band tends to cut corners. ${ }^{46}$ Only using certain parts of the gradient partly mitigates these effects, leading to an effective gradient ${ }^{45}$

$$
\mathbf{g}_{\mathrm{NEB}}=\mathbf{g}^{\perp}+\mathbf{g}_{\mathrm{spr}}^{\|} .
$$

This approach is the nudged elastic band (NEB) method, where the component of the true gradient parallel to the path is removed, and the component of the spring gradient perpendicular to the path is projected out. ${ }^{46,48}$

In some cases, faster convergence around the images corresponding to local maxima may be achieved by retaining part of the perpendicular component of the spring gradient, yielding a doubly-nudged elastic band (DNEB) ${ }^{49}$ with gradient

$$
\mathbf{g}_{\text {DNEB }}=\mathbf{g}^{\perp}+\mathbf{g}_{\text {spr }}^{\|}+\hat{\mathbf{g}}^{\perp}-\left(\mathbf{g}_{\text {spr }}^{\perp} \cdot \hat{\mathbf{g}}^{\perp}\right) \hat{\mathbf{g}}^{\perp} .
$$

In the present work we employ a custom L-BFGS ${ }^{54,55}$ minimiser for both NEB and DNEB, with all images optimised simultaneiously. The optimisation was terminated when the RMS force on the band is below $10^{-3} \mathrm{kcal} \mathrm{mol}^{-1} \AA^{-1}$, or 4000 minimisation steps had been taken. We chose a large L-BFGS history size (1000 steps), as we did not attempt to optimise run times.

\section{A. Quasi-continuous interpolation}

Any interpolation scheme may be chosen to create the initial set of discrete images, and a linear interpolation is often sufficient for simple mechanisms. However, for complex structural rearrangements of biomolecules, unphysical chain crossings can arise from the use of discrete images. This problem can be mitigated using quasi-continuous interpolation $(\mathrm{QCI}),{ }^{56,57}$ which retains a discrete representation of the pathway.

QCI employs a simple auxiliary potential, containing attractive constraints and Coulombic repulsions with a relatively short cutoff for interactions. The attractive constraints (bonds) can easily be identified for biomolecules based on sequence, and further constraints, to preserve chirality and prevent cistrans isomerism, can be added. The key feature of the auxiliary potential is the final component, a repulsive term for any local minima in the distance between pairs of atoms in adjacent images. This repulsion corresponds to the worstcase energetic contribution, which helps to prevent chaincrossing, and represents the quasi-continuous part of the potential. The details of the interpolation may be found in previous reports, ${ }^{56,57}$ where we have shown that it can produce a more physical, low-energy set of images.

\section{B. Internal coordinate interpolations}

Internal coordinate transformations can sometimes produce better initial guess interpolations for anisotropic potentials with bond angle and dihedral angle terms,$^{58}$ as they reduce higher order coupling between coordinates ${ }^{59,60}$ Furthermore, the use of internal coordinates permits larger optimisation steps, but the additional coordinate transforms required can make the overall procedure less efficient. Previous work showed that an efficiency gain is possible for smaller peptides, while for large systems Cartesian coordinates are faster. ${ }^{61} \mathrm{We}$ tested these internal coordinates schemes in the present work, to assess their capability to help with pathways that exhibit internal minima and very different energy scales.

\section{Redistribution of images and adjusting the spring constant}

One straightforward adaption of the NEB and DNEB algorithms is to correct the image spacing at intervals during the optimisation. As nudging does not guarantee a good spacing between images, subtle features on the pathway may not be represented well in a single interpolation. Clearly, repeated connection attempts between newly discovered local minima should eventually yield a connected path, but improved initial interpolations may provide a more efficient solution.

In selected runs we tested redistribution of the images at regular intervals in the NEB or DNEB refinement. The Euclidean distance between successive images was calculated, and the images were redistributed by simply shifting them along the straight line segments to give equal distances along the original interpolation.

We also adjusted the spring constant in some NEB and DNEB runs throughout the band refinement. The OPTIM program includes alternative schemes for dynamic spring constant adjustment. One of these increases or decreases $k_{\mathrm{spr}}$ by a small fraction depending on whether the standard deviation of the image separation relative to the average is above or below a specified tolerance. However, we found that a schedule specifying initial (larger) and final (smaller) values of $k_{\mathrm{spr}}$ with a steady decrease determined by the maximum number of refinement iterations, worked better for the problem in question. All the results we present below are based on this protocol. We note that alternative procedures based on NEB and comparisons with other schemes have also been presented before. ${ }^{62-66}$ In the present report we focus on the NEB and DNEB schemes, which probably account for most transition state and pathway investigations in the literature. 
TABLE I: The five stationary points forming the target discrete path for the $\tau$ peptide. The endpoints used for starting the interpolation algorithms are Min 1 and Min 3.

\begin{tabular}{lcc}
\hline \hline Stationary point & $\mathrm{E}\left(\mathrm{kcal} / \mathrm{mol}^{-1}\right)$ & $\mathrm{F}\left(\mathrm{kcal} / \mathrm{mol}^{-1}\right)^{\mathrm{a}}$ \\
\hline Min 1 & $-1038.604929(2)$ & $1.506143(2)$ \\
TS 1 & $-1037.031021(2)$ & $2.942694(4)$ \\
Min 2 & $-1040.994529(3)$ & $0.000000(0)$ \\
TS 2 & $-1036.440185(1)$ & $2.314330(3)$ \\
Min 3 & $-1037.169948(0)$ & $1.658213(7)$ \\
\hline \hline
\end{tabular}

${ }^{\text {a }}$ Free energies calculated at $310 \mathrm{~K}$ within the harmonic superposition approximation; all values are relative to the lowest energy stationary point (Min 2).

\section{SYSTEM SETUP}

We have observed problems with pathways involving multiple steps and widely different barriers in previous work for atomic and molecular clusters, proteins, nucleic acids, coarsegrained models of mesoscopic systems, and in condensed matter. The difficulties that arise when trying to describe barriers on different energy and length scales in the same interpolation seem to be generic, and we have therefore analysed one representative system in detail. The choice of a peptide enabled us to compare additional initial interpolation schemes that exploit internal coordinates, to see if these offer any advantage in a case where they appear to be well suited to describing the motion in question.

\section{A. A two-step peptide rearrangement}

The example we have chosen to illustrate the issue with interpolation for rearrangements involving intermediate minima is for a $\tau$ protein peptide fragment of 446 atoms with a 27 amino acid sequence (N to C) KVQII-NKKLD-LSNVQSKCGS-KDNIK-HV.

The pathway in question is a rearrangement of the $\mathrm{N}$ terminal lysine via a rotation of the side chain, which involves a low-energy intermediate minimum. The shortest discrete pathway contains only three minima, which are shown in Fig. 1, and the potential and (harmonic) free energies of all stationary points on this discrete path are given in Table I. The initial distance between minimum 1 and minimum 3 is $5.77 \AA$ after optimal spatial and permutational alignment, ${ }^{67}$ and the shortest discrete path has a length of $22.50 \AA$ (11.51 $\AA$ between minima 1 and 2, and $10.99 \AA$ between minima 2 and 3).

\section{B. Simulation setup}

We used the NEB and DNEB implementations in the OPTIM $^{68}$ program to connect the two endpoints. The set of simulations included four different sets for NEB and DNEB
TABLE II: Parameters used in the DNEB and NEB runs. In each case only one iteration was allowed for the

double-ended search, with up to 4000 minimisation steps for the band optimisation and a convergence criterion of $0.001 \mathrm{kcal} \mathrm{mol}^{-1} \AA^{-1}$.

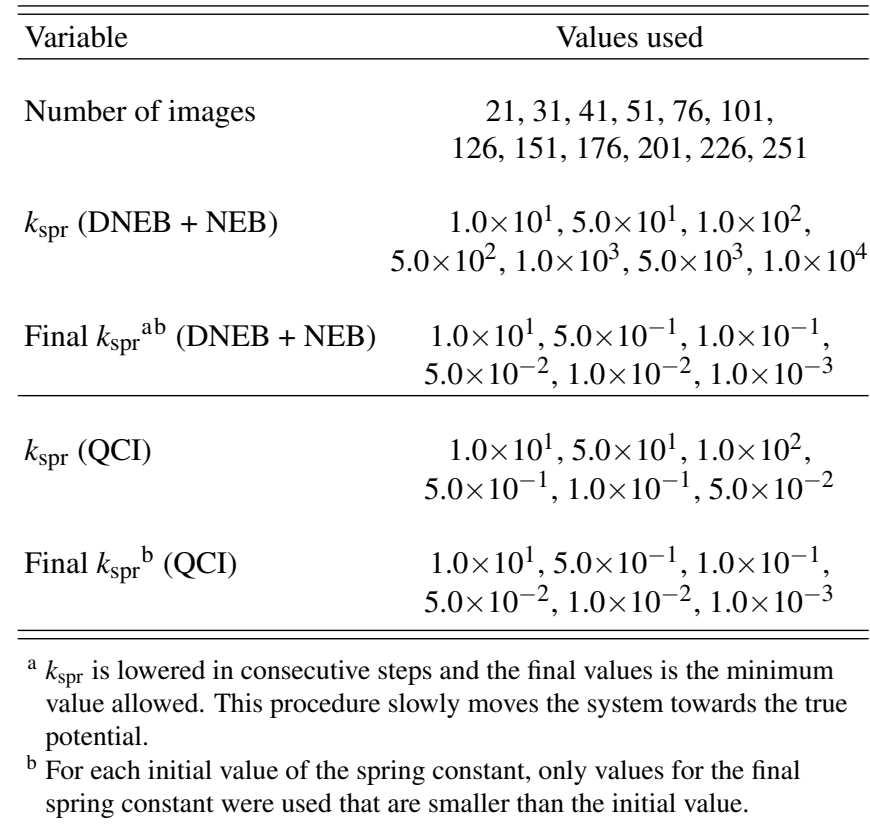

formulations: (set 1) an initial linear interpolation, with redistribution of the images and adjustment of the spring constant; (set 2) with only the redistribution; (set 3) with only the adjustment; (set 4) without redistribution or adjustment. We further tested QCI as a different initial interpolation, followed by optimisation using the DNEB gradient (QCI+DNEB), including runs with image redistribution and spring constant adjustments. The QCI+DNEB simulations also employ springs to keep the images connected, with a separate spring constant parameter. We chose to use the value at the end of the QCI process as the initial value for the DNEB band optimisation. This choice limits the useful range of spring constants somewhat, but is self-consistent. In addition, we tested initial internal coordinate interpolations with DNEB, as described below. We used the ff14SB force field ${ }^{69}$ with implicit solvent $(\text { igb2 })^{70-72}$ in AMBER $12^{73}$ throughout. For the QCI+DNEB runs, cistrans constraints were employed. ${ }^{57}$ The different sets of simulation parameters, namely the number of images for the interpolation, and the spring constants employed, are given in Table II.

For the internal coordinate interpolations the following options were tested. An interpolation in natural internal coordinates was used, with and without permutational alignment of torsional angles, and the interpolation images were initially placed at equal separations along the arclength. Another set of connections with permutational alignment of the torsions was considered, where the internal coordinate images are used directly in the band optimisation, without attempting to space them equally along the path. Additionally, we considered connections where both a Cartesian and an internal co- 


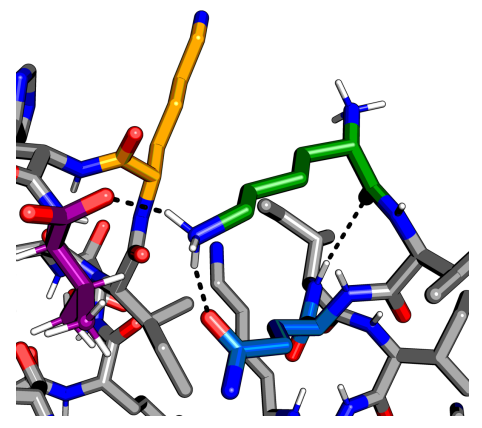

(a) Minimum 1

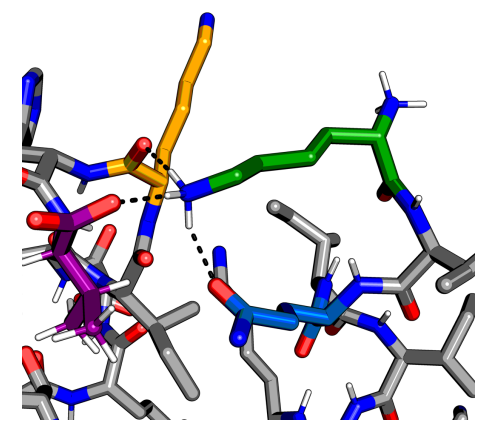

(b) Minimum 2

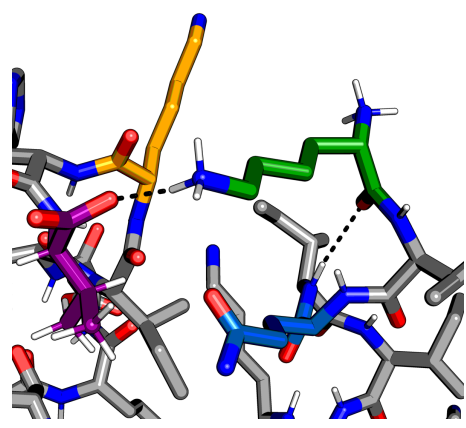

(c) Minimum 3

FIG. 1: The three minima on the target discrete path considered in this study. The transition involves a rotation of the side chain of the N-terminal Lys1 (green), losing contact with Gln3 (blue). The contact with the C-terminal Val27 (purple) remains throughout, and the intermediate minimum stabilises the transition through a new contact formed between Lys1 and the backbone of Lys 25 (orange).

ordinate interpolation were constructed and the lower energy band was then chosen. Detailed descriptions of these schemes can be found elsewhere. ${ }^{58,61}$ Our tests did not reveal any significant advantage for initial internal coordinate interpolation. Furthermore, previous work indicates the computational costs are higher than for Cartesian coordinates, especially for larger systems. We therefore omit details of these calculations for brevity.

\section{RESULTS AND DISCUSSION}

In total, we ran 2,760 connection attempts, each limited to one doubled-ended search, with 1,152 runs for both DNEB and NEB, and another 456 using QCI and DNEB (QCI+DNEB). The NEB/DNEB runs each include 84 tests with a fixed spring constant for runs with (set 3 ) and without (set 4) redistributing the images, and 492 tests adjusting the spring constants with (set 1) and without (set 2) image redistribution. For QCI+DNEB, we tested 72 parameter combinations without spring constant adjustments, with and without redistributing images, and another 312 runs adjusting the spring constant, but without redistribution. We allowed for a maximum wall time of 6 days for every calculation (with the fastest runs completing in 30 minutes on a single core on a six-core dual Xeon X5650 (2.6GHz) processor),${ }^{74}$ meaning that we allowed runs to complete that were up to $288 \times$ slower than the fastest ones. All DNEB and all QCI+DNEB runs finished well within this window, however, eight of the NEB attempts did not, and are therefore excluded from the analysis.

Broadly speaking, the results for the interpolation attempts fall into two categories: in the first set, we observe a band with a single transition state candidate, and subsequent refinement therefore only leads to one transition state. In the second set, more candidates are found, usually in much longer interpolations, and a single cycle can produce a complete discrete path between the endpoints. Two example bands are shown in Fig. 2. We note that the band shown in red includes a rel-

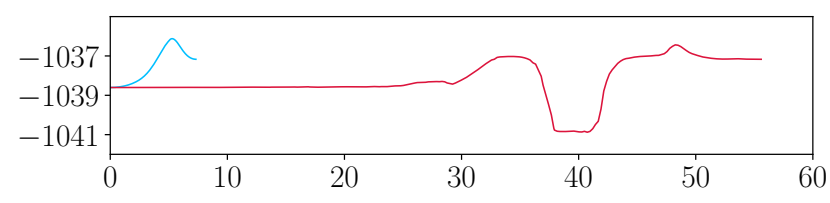

FIG. 2: Comparison of two different interpolations using DNEB after convergence or the iteration limit is reached, with the band length (sum of segments) in $\AA$ on the horizontal axis, while the vertical axis is the potential energy in $\mathrm{kcal} / \mathrm{mol}^{-1}$. In the first set of results, we see a short band (approx. $8 \AA$ ) with only a single maximum (blue). In the second set, the band (red) is much longer (around $55 \AA$ ) and it contains an intervening low-energy state with transition state candidates on either side. 251 images were used in both runs, with an initial spring constant of $5.0 \times 10^{3} \mathrm{kcal} \mathrm{mol}^{-1} \AA^{-2}$.

For the short blue band, no redistribution or spring adjustments were allowed. For the longer band, the images were redistributed and the spring constant allowed to relax to a final value of $5.0 \times 10^{-3} \mathrm{kcal} \mathrm{mol}^{-1} \AA^{-2}$.

atively flat region at the beginning, likely due to an overall rotation that results from the initial alignment. If this motion were removed, the band would nevertheless be much longer.

For the computations returning only a single transition state, we observe a short interpolation path, with relatively little change compared to the initial interpolation, and only one maximum in the profile. However, in the bands that locate more transition states, even if they start from similar interpolations (compare Fig. 2 with the top panel Fig. 3), the band stretches and more features appear, as shown in Fig. 3.

\section{A. DNEB interpolations}

In the four different sets of calculations, we only obtain fully connected paths when either the images are redistributed 


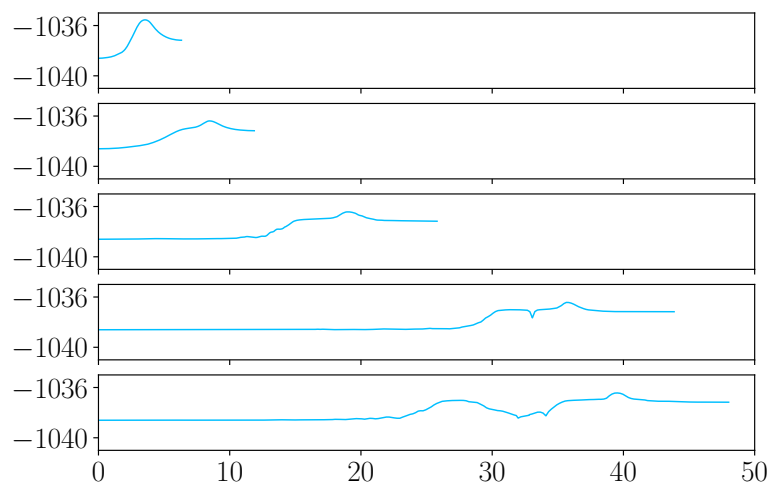

FIG. 3: Evolution of the band for a DNEB run with redistribution of images every hundred steps, showing the slowly increasing length of the band and the location of an additional internal minimum emerging. The run shown is the red band from Fig. 2, i.e. 251 images with redistributions and spring constant adjustment.

and the spring constant is adjusted, or if only the spring constant is altered. For the former set there are 173 successful attempts, and for the latter, 8. Clearly, adjusting the spring constant, allowing the path to gradually extend, helps to produce a better representation of the shortest discrete path in the interpolation, and this approach can be improved by additional redistribution of images, which on its own is not as effective. We observe no runs with more than one transition state located in the other two sets ( 2 and 4$)$, and the final length of the interpolations is very similar (set 2: $8.56 \pm 2.43 \AA$; set 4 : $8.50 \pm 2.37 \AA$ ). Unsuccessful runs in the other two sets, where no complete discrete path between the endpoints was found, can also exhibit relatively long paths, and multiple transition states are located for a number of them. Overall, 282 attempts in set 1 and 174 for set 3 located more than one transition state, with the maximum number being 29 and 27, respectively. The average numbers are $8.4 \pm 5.9$ and $3.8 \pm 3.1$ transition states for set 1 and set 3 for the connection attempts that locate more than one transition state. This increase in the number of stationary points is correlated with the length of the final interpolation, with an average value for set 1 of $30.9 \AA$, and $22.9 \AA$ in set 3 . In both cases the maximum sum of distances between successive images was greater than $100 \AA$. While it is necessary to have a longer path than the initial, linear distance between the minima, if the band becomes too long, it meanders around the actual region of interest. While this behaviour is undesirable for initial connection attempts to create a network, it may provide an interesting alternative for additional sampling at later stages of the landscape exploration.

\section{Key parameters for successful DNEB interpolations}

From these results we see that dynamic adjustment of the spring constant, which allows for longer paths, significantly
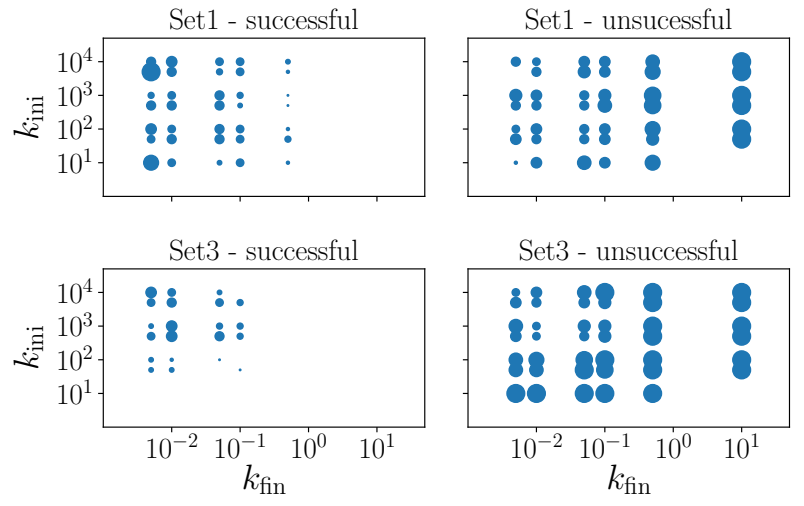

FIG. 4: An overview of the successful (left) and unsuccessful attempts using DNEB (right) for sets 1 (top) and 3 (bottom), for the various initial (y-axis) and final (x-axis) for the spring constant. The size of each data point represents the squared number of attempts with the given parameters. We see that a large ratio of final to initial spring constants helps to produce successful connection attempts.

improves the performance of the double-ended searches. This observation indicates that it takes a longer interpolation with enough images to follow the actual path accurately enough to identify some of the stationary points. The first lesson to be learnt is that an interpolation path may not be a good representation of the required pathway between structures, even if it appears to be converged. Missing features, especially lowlying internal minima, could have significant effects on observable properties, especially predictions of interconversion rates.

While the resulting trends are clear, there is a further question, namely, which parameter variations are actually key to producing successful runs. We divide sets 1 and 3 into two subsets, each with the successful runs in one, and the unsuccessful attempts in the other. As the three input parameters are the initial and the final spring constants, as well as the number of images allowed in the interpolation, we can first of all test for differences in these parameters between the subsets.

In both sets 1 and 3, we observe that the initial spring constant is larger in the successful runs. However the differences are small, and, apart from the lowest value for the initial spring constant in set 3, we observe the full range of values in the successful and unsuccessful runs.

In contrast, the average final spring constant in the successful subsets is about two orders of magnitude smaller, and only the smaller values of the final spring constant resulted in successful connection attempts. These trends are illustrated in Fig. 4, showing that for larger final values of the spring constant, only a small number of attempts are successful. Redistribution of the images, in addition to spring adjustments, allows for smaller differences between the initial and final values, both with smaller initial and larger final values, compared to no redistribution of images.

The number of images is also important. However, it is in- 
teresting that a good choice of values for the initial and final spring constants allows for interpolations with a smaller number of images to be successful as well (see Fig. 5). This is a useful observation, because a high image density requires a significant amount of memory for large systems, especially when a long history length is desirable in the LBFGS procedure.

\section{Quasi-continuous interpolation}

For QCI+DNEB, we only considered parameter sets 2, 3 and 4; and obtained the full discrete path for 10 runs in set 2 and for 19 attempts in set 3. Again, no attempt was successful without using image redistribution or adjustments to the spring constant. In contrast to DNEB with a linear interpolation, we obtained successful connections for set 2, but the fraction of successful attempts in set 2 and set 3 using QCI is lower than for sets 1 and 3 with DNEB alone (0.14 and 0.06 vs. 0.35 and 0.18 ). As we started the QCI+DNEB runs with a set of smaller initial values for the spring constant this observation is in line with the observations above. The average path length for all sets increases compared to DNEB with a linear interpolation (set 2: $21.3 \AA$, set 3: $23.2 \AA$, set 4: $13.6 \AA$ ).

Looking more closely at the subsets of successful and unsuccessful runs for sets 2 and 3 , enables us to identify trends, as above. For set 3 , we find again that lower final values for the spring constant are more likely to result in successful connection attempts. However, not only large differences in the initial and final values, but also the smallest possible differences in the spring constants, can result in full discrete paths. As the construction of the interpolation in QCI contains an adjustment of the spring constants as well, we see that a larger spring constant adjustment is required for flexibility, but this adjustment can occur in the initial stages when the interpolation is constructed, or during the band optimisation. This point is supported by the fact that for the runs with image redistribution and fixed spring constant, we only observe successful attempts for the smallest values of the spring constant. Furthermore, the tendency for successful runs to have a larger number of images is again observed, but the better the choice of values for the spring constant, the smaller the number of images can be. Both these features are shown in Fig. 6.

\section{B. NEB interpolations}

Using the NEB gradient, we obtain a different picture from DNEB. Runs with spring constant adjustments were all unsuccessful, and, in contrast, there were 51 successful attempts for set 4 and 31 for set 2 . The number of transition states found is higher than for DNEB for the successful interpolations, with $16.6 \pm 12.1$ for set 2 and $9.3 \pm 6.3$ for set 4 . The average path length is also significantly increased (set $2: 77.1 \AA$, set 4: $53.7 \AA$ ) with a maximum length of over $300 \AA$. If we allow for a redistribution of images, the value of the spring constant is not significant, but a larger number of images increases the chance of success. If the images are not redistributed the op- posite trend is observed, and the number of images is less significant, but a lower spring constant is more likely to result in a connected path.

Clearly, the different projections of the gradient affect the paths significantly, and also the possibilities for improvements. One observation emerging from this analysis is that the NEB bands are longer than DNEB bands, as expected from the additional gradient components in the DNEB formulation. While this feature can improve the interpolation, it also significantly increases the computational time required. It also highlights that the actual interpolation obtained may deviate from true discrete paths, and depends significantly on the simulation parameters used.

\section{CONCLUSION}

In this study we have investigated the nudged and doublynudged elastic band interpolations, and their dependence on input parameters, in characterising the path between two local minima for a peptide with 27 residues. The first important conclusion is that the resulting interpolation bands depend strongly on the input parameters, and may not reproduce all the transition states and local minima on the path.

For the DNEB interpolations we find that systematically decreasing the spring constants during the refinement process helps to improve the final band, gradually lowering the spring component of the total energy. This scheme can be further improved when the images are periodically redistributed along the segments of the interpolation band between successive images. The number of images is less important if a large initial and a small final value of the force constant are used, allowing for a reduced memory requirement.

The use of quasi-continuous interpolation ${ }^{56,57}$ with DNEB provides an alternative way to find fully connected paths effectively. Importantly, the use of QCI+DNEB not only yields lower energy paths, as previously reported ${ }^{57}$ but in addition, spring adjustment from a large initial to a small final value improves the results.

Finally, we observe that NEB interpolations do not seem to benefit from these adjustments, as the gradient is constructed differently. To improve the interpolations a longer path is desirable, allowing for the location of all relevant stationary points. Such a path may be achieved using smaller values for the spring constants or a larger number of images.

Overall, we note that a careful adjustment of input parameters can significantly improve double-ended interpolation schemes, but the interpolations themselves do not necessarily represent the structural transitions faithfully. We recommend that NEB or DNEB interpolations are optimised until the local maxima are clear. ${ }^{49,53}$ These images should then be used as starting points for accurate single-ended refinement using hybrid eigenvector-following, ${ }^{51,52}$ and the connectivity should be checked by calculating approximate steepest-descent paths. If a complete connection does not result, the procedure can be repeated using the missing connection algorithm ${ }^{75}$ to select new pairs of minima to connect.

Finally, it is also important to realise that a successful ini- 

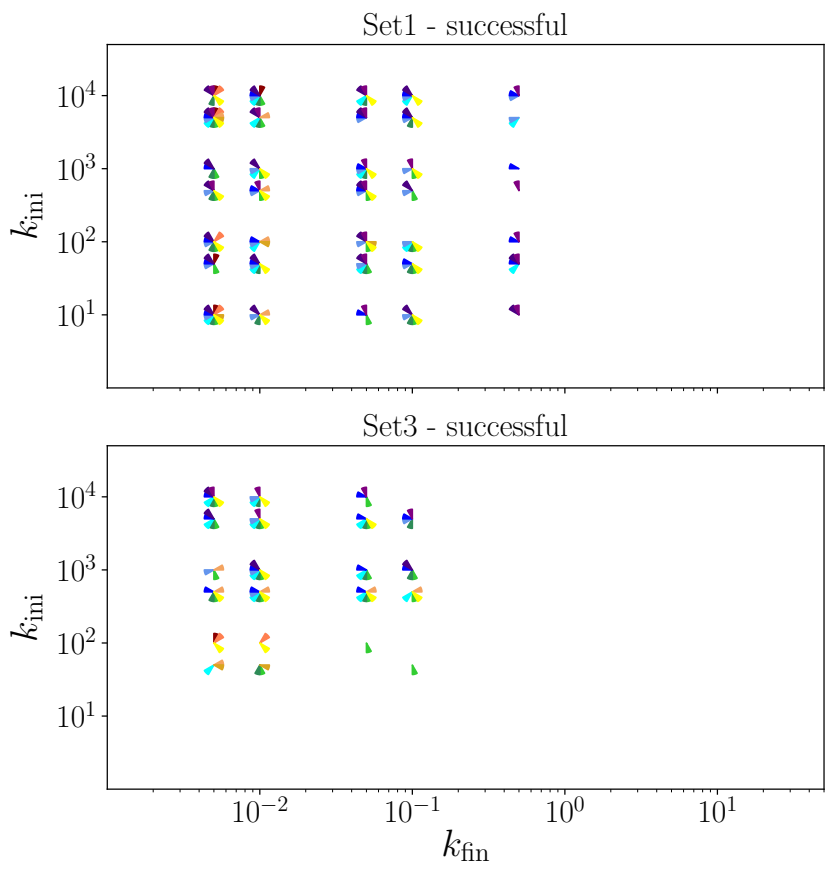

Set1 - unsucessful

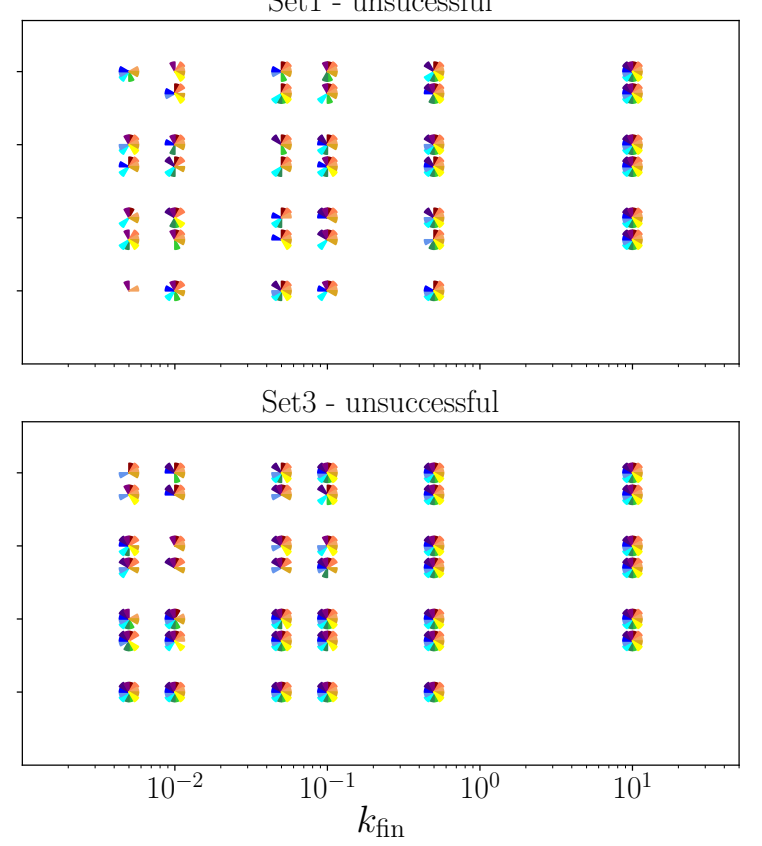

FIG. 5: Summary of the effect of variations in the spring constant and the number of images for test sets 1 and 3 for the DNEB simulations. The coloured wheels represent the different number of images going clockwise from lowest (first sector, 12 to 1 o'clock, 21 images) up to the highest number (11 to 12 o'clock, 251 images). We see that a larger number of images is especially useful if the change in the spring constants is small.

tial connected path may be kinetically irrelevant. For complex transitions, involving tens or hundreds of transition states, the initial path is simply a starting point for refinement of a kinetic transition network (KTN). ${ }^{43,76-78}$ The discrete path sampling approach $^{12,13}$ subsumes a variety of procedures designed to converge $^{79}$ the phenomenological rate constant of interest between specified product and reactant states. For example, the PATHSAMPLE program ${ }^{80}$ includes schemes that aim to reduce path length, locate lower barrier paths, and remove artificial kinetic traps in the network. ${ }^{75,81,82}$ All of these procedures are based on double-ended searches, analogous to the initial pathway search, but with different target endpoint minima. All the stationary points found in unsuccessful connection attempts can be added to the database, and employed in the next round of searches, where the gaps that may need to be bridged should be smaller. One of the longest pathways we have addressed, involving conformational changes associated with membrane fusion in influenza A haemagglutinin, initially required over 4,500 transition states. On database refinement, which we will describe elsewhere, the fastest pathways involve around 3,500 steps. Our observation is that initial paths with a few hundred transition states or more usually become at least $25 \%$ shorter (and much faster) on database refinement.

\section{ACKNOWLEDGMENTS}

KR is supported by a Henslow Research Fellowship from the Cambridge Philosophical Society and Robinson College,
DJW acknowledges support by the EPSRC.

\section{DATA AVAILABILITY}

The data that support the findings of this study are openly available at Zenodo https://doi.org/10.5281/zenodo.3885467. ${ }^{83}$ This example will also be added to the OptBench web site at http://optbench.org/

${ }^{1}$ D. J. Wales, Energy Landscapes (Cambridge University Press, Cambridge, 2003).

${ }^{2}$ C. M. Dobson and M. Karplus, "The fundamentals of protein folding: bringing together theory and experiment," Curr. Opin. Struc. Biol. 9, 92101 (1999).

${ }^{3}$ P. G. Wolynes, "Energy landscapes and solved protein-folding problems," Philos. T. Roy. Soc. A 363, 453-467 (2005).

${ }^{4}$ K. A. Dill, S. B. Ozkan, M. S. Shell, and T. R. Weikl, "The protein folding problem,” Annu. Rev. Biophys. 37, 289-316 (2008).

${ }^{5}$ P. G. Wolynes, W. A. Eaton, and A. R. Fersht, "Chemical physics of protein folding," Proc. Natl Acad. Sci. USA 109, 17770-17771 (2012).

${ }^{6}$ D. J. Wales and P. Salamon, "Observation time scale, free-energy landscapes, and molecular symmetry," Proc. Natl. Acad. Sci. USA 111, 617 622 (2014).

${ }^{7}$ L. R. Pratt, "A statistical method for identifying transition states in high dimensional problems," J. Chem. Phys. 85, 5045-5048 (1986).

${ }^{8}$ C. Dellago, P. G. Bolhuis, F. S. Csajka, and D. Chandler, "Transition path sampling and the calculation of rate constants," J. Chem. Phys. 108, 19641977 (1998).

${ }^{9}$ C. Dellago, P. G. Bolhuis, and D. Chandler, "On the calculation of reaction rate constants in the transition path ensemble," J. Chem. Phys. 110, 66176625 (1999). 

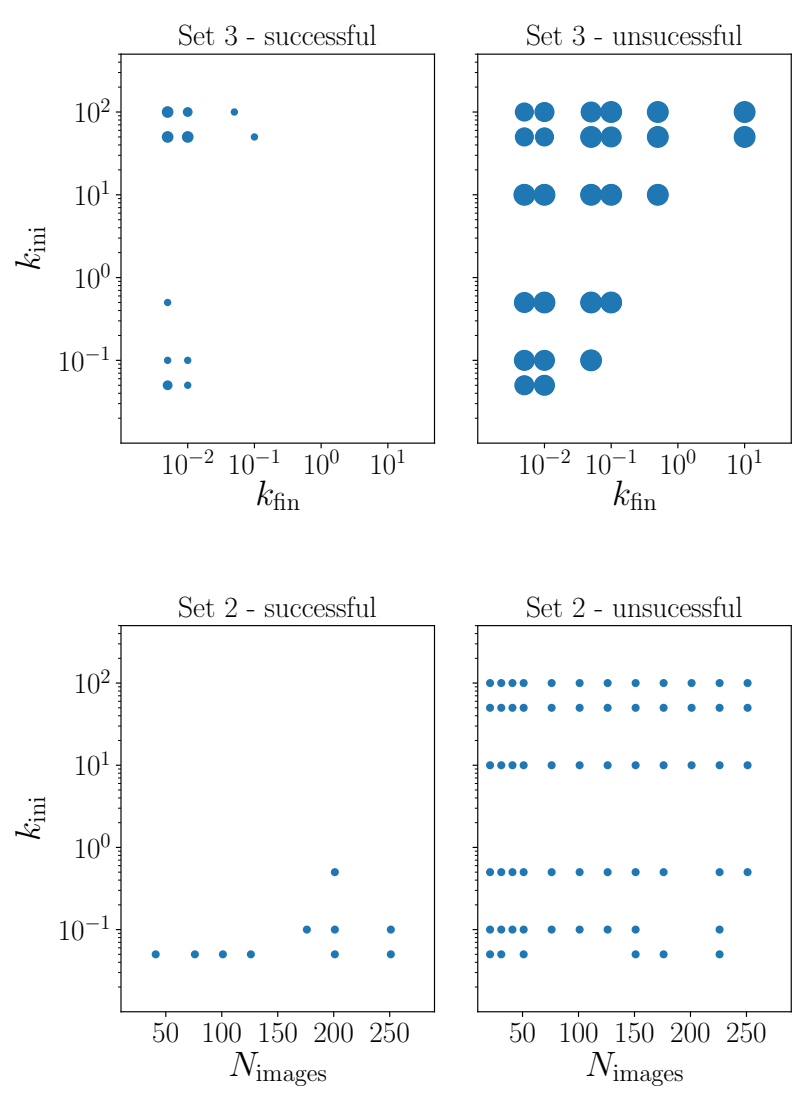

FIG. 6: Overview of the results for the runs using QCI+DNEB. Top: a plot of the initial and final spring constants (as in Fig. 4 for DNEB), showing the two distinct sets of successful runs. Bottom: dependence of the successful outcomes on the number of images and the initial spring constant, if QCI+DNEB is used with image redistribution. The smaller initial values of the spring constant, allowing for relaxation in the interpolation constructions, exhibit some success, while the change in the number of images has a smaller effect.

${ }^{10}$ P. G. Bolhuis, D. Chandler, C. Dellago, and P. L. Geissler, "Transition path sampling : Throwing ropes over rough mountain passes, in the dark," Annu. Rev. Phys. Chem. 53, 291-318 (2002).

${ }^{11}$ C. Dellago and P. G. Bolhuis, "Transition path sampling and other advanced simulation techniques for rare events," Adv. Polymer Sci. 221, 167-233 (2009).

${ }^{12}$ D. J. Wales, "Discrete path sampling," Mol. Phys. 100, 3285-3305 (2002).

${ }^{13}$ D. J. Wales, "Some further applications of discrete path sampling to cluster isomerization,” Mol. Phys. 102, 891-908 (2004).

${ }^{14}$ T. S. van Erp, D. Moroni, and P. G. Bolhuis, "A novel path sampling method for the calculation of rate constants," J. Chem. Phys. 118, 77627774 (2003)

${ }^{15}$ T. S. van Erp and P. G. Bolhuis, "Elaborating transition interface sampling methods," J. Comput. Phys. 205, 157-181 (2005)

${ }^{16}$ A. K. Faradjian and R. Elber, "Computing time scales from reaction coordinates by milestoning," J. Chem. Phys. 120, 10880-10889 (2004).

${ }^{17}$ D. Moroni, T. S. van Erp, and P. G. Bolhuis, "Investigating rare events by transition interface sampling," Phys. A Stat. Mech. its Appl. 340, 395-401 (2004).
${ }^{18}$ G. A. Huber and S. Kim, "Weighted-ensemble brownian dynamics simulations for protein association reactions," Biophys. J. 70, 97-110 (1996).

${ }^{19}$ B. W. Zhang, D. Jasnow, and D. M. Zuckerman, "The "weighted ensemble" path sampling method is statistically exact for a broad class of stochastic processes and binning procedures," J. Chem. Phys. 132, 054107 (2010).

${ }^{20}$ L. T. Chong, A. S. Saglam, and D. M. Zuckerman, "Path-sampling strategies for simulating rare events in biomolecular systems," Curr. Opin. Struct. Biol. 43, 88-94 (2017).

${ }^{21}$ R. J. Allen, P. B. Warren, and P. R. ten Wolde, "Sampling rare switching events in biochemical networks," Phys. Rev. Lett. 94, 018104 (2005).

${ }^{22}$ R. J. Allen, D. Frenkel, and P. R. t. Wolde, "Simulating rare events in equilibrium or nonequilibrium stochastic systems," J. Chem. Phys. 124, 024102 (2006).

${ }^{23}$ R. J. Allen, D. Frenkel, and P. R. t. Wolde, "Forward flux sampling-type schemes for simulating rare events: Efficiency analysis," J. Chem. Phys. 124, 194111 (2006).

${ }^{24}$ A. M. A. West, R. Elber, and D. Shalloway, "Extending molecular dynamics time scales with milestoning: Example of complex kinetics in a solvated peptide," J. Chem. Phys. 126, 145104 (2007).

${ }^{25}$ E. Vanden-Eijnden, M. Venturoli, G. Ciccotti, and R. Elber, "On the assumptions underlying milestoning," J. Chem. Phys. 129, 174102 (2008).

${ }^{26}$ J. M. Bello-Rivas and R. Elber, "Exact milestoning," J. Chem. Phys. 142, 094102 (2015).

${ }^{27}$ F. Noe, C. Schutte, E. Vanden-Eijnden, L. Reich, and T. R. Weikl, "Constructing the equilibrium ensemble of folding pathways from short offequilibrium simulations," Proc. Natl. Acad. Sci. USA 106, 19011-19016 (2009).

${ }^{28}$ V. S. Pande, K. Beauchamp, and G. R. Bowman, "Everything you wanted to know about Markov State Models but were afraid to ask," Methods 52, 99-105 (2010).

${ }^{29}$ T. J. Lane, D. Shukla, K. A. Beauchamp, and V. S. Pande, "To milliseconds and beyond: challenges in the simulation of protein folding," Curr. Opin. Struct. Biol. 23, 58-65 (2013).

${ }^{30}$ J. D. Chodera and F. Noé, "Markov state models of biomolecular conformational dynamics," Curr. Opin. Struct. Biol. 25, 135-144 (2014).

${ }^{31}$ B. E. Husic and V. S. Pande, "Markov State Models: From an art to a science," J. Am. Chem. Soc. 140, 2386-2396 (2018).

${ }^{32}$ W. Ren, E. Vanden-Eijnden, P. Maragakis, and W. E, "Transition pathways in complex systems: Application of the finite-temperature string method to the alanine dipeptide," J. Chem. Phys. 123, 134109 (2005).

${ }^{33}$ J. A. Joseph, K. Röder, D. Chakraborty, R. G. Mantell, and D. J. Wales, "Exploring biomolecular energy landscapes," Chem. Commun. 53, 69746988 (2017).

${ }^{34}$ K. Röder, J. A. Joseph, B. E. Husic, and D. J. Wales, "Energy landscapes for proteins: From single funnels to multifunctional systems," Adv. Theory Simul. 2, 1800175 (2019).

${ }^{35}$ D. J. Wales, "Exploring energy landscapes," Ann. Rev. Phys. Chem. 69, 401-425 (2018).

${ }^{36}$ Y. Sugita and Y. Okamoto, "Replica-exchange molecular dynamics method for protein folding," Chem. Phys. Lett. 314, 141-151 (1999).

${ }^{37}$ G. Torrie and J. Valleau, "Nonphysical sampling distributions in Monte Carlo free-energy estimation: Umbrella sampling," J. Comput. Phys. 23, 187-199 (1977).

${ }^{38}$ A. Laio and M. Parrinello, "Escaping free-energy minima." Proc. Natl. Acad. Sci. USA 99, 12562-12566 (2002).

${ }^{39}$ A. F. Voter, "Hyperdynamics: Accelerated molecular dynamics of infrequent events," Phys. Rev. Lett. 78, 3908-3911 (1997).

${ }^{40}$ S. V. Krivov and M. Karplus, "Hidden complexity of free energy surfaces for peptide (protein) folding," Proc. Nat. Acad. Sci. USA 101, 14766-14770 (2004).

${ }^{41}$ S. V. Krivov and M. Karplus, "One-dimensional free-energy profiles of complex systems: progress variables that preserve the barriers." J. Phys. Chem. B 110, 12689-12698 (2006).

${ }^{42}$ S. V. Krivov and M. Karplus, "Diffusive reaction dynamics on invariant free energy profiles." Proc. Nat. Acad. Sci. USA 105, 13841-13846 (2008).

${ }^{43}$ F. Noé and S. Fischer, "Transition networks for modeling the kinetics of conformational change in macromolecules," Curr. Opin. Struc. Biol. 18, 154-162 (2008).

${ }^{44}$ N. G. van Kampen, Stochastic Processes in Physics and Chemistry (NorthHolland, Amsterdam, 1981). 
${ }^{45}$ G. Mills, H. Jónsson, and G. K. Schenter, "Reversible work transition state theory: Application to dissociative adsorption of hydrogen," Surf. Sci. 324, 305-337 (1995).

${ }^{46}$ H. Jónsson, G. Mills, and K. Jacobsen, "Nudged elastic band method for finding minimum energy paths of transitions," in Classical and quantum dynamics in condensed phase simulations, edited by G. C. B. J. Berne and D. F. Coker (World Scientific, 1998) pp. 385-404.

${ }^{47}$ G. Henkelman, B. P. Uberuaga, and H. Jónsson, "A climbing image nudged elastic band method for finding saddle points and minimum energy paths," J. Chem. Phys. 113, 9901-9904 (2000).

${ }^{48} \mathrm{G}$. Henkelman and H. Jónsson, "Improved tangent estimate in the nudged elastic band method for finding minimum energy paths and saddle points," J. Chem. Phys. 113, 9978-9985 (2000).

${ }^{49}$ S. A. Trygubenko and D. J. Wales, "A doubly nudged elastic band method for finding transition states," J. Chem. Phys. 120, 2082-2094 (2004).

${ }^{50}$ D. Sheppard, R. Terrell, and G. Henkelman, "Optimization methods for finding minimum energy paths," J. Chem. Phys. 128, 134106 (2008).

${ }^{51} \mathrm{G}$. Henkelman and H. Jónsson, "A dimer method for finding saddle points on high dimensional potential surfaces using only first derivatives," J. Chem. Phys. 111, 7010-7022 (1999).

${ }^{52}$ L. J. Munro and D. J. Wales, "Defect migration in crystalline silicon," Phys. Rev. B 59, 3969-3980 (1999).

${ }^{53}$ Y. Zeng, P. Xiao, and G. Henkelman, "Unification of algorithms for minimum mode optimization,” J. Chem. Phys. 140, 044115 (2014).

${ }^{54}$ J. Nocedal, "Updating quasi-newton matrices with limited storage," Math. Comput. 35, 773-782 (1980).

${ }^{55}$ D. Liu and J. Nocedal, "On the limited memory bfgs method for large scale optimization,” Math. Prog. 45, 503 (1989).

${ }^{56}$ D. J. Wales and J. M. Carr, "Quasi-continuous interpolation scheme for pathways between distant configurations," J. Chem. Theory Comput. 8, 5020-5034 (2012).

${ }^{57}$ K. Röder and D. J. Wales, "Predicting pathways between distant configurations for biomolecules," J. Chem. Theory Comput. 14, 4271-4278 (2018).

${ }^{58}$ M. S. Bauer, B. Strodel, S. N. Fejer, E. F. Koslover, and D. J. Wales, "Interpolation schemes for peptide rearrangements," J. Chem. Phys. 132, 054101 (2010).

${ }^{59}$ B. Paizs, J. Baker, S. Suhai, and P. Pulay, "Geometry optimization of large biomolecules in redundant internal coordinates," J. Chem. Phys. 113, 6566 (2000).

${ }^{60}$ G. Fogarasi, X. Zhou, P. W. Taylor, and P. Pulay, "The calculation of ab initio molecular geometries: Efficient optimization by natural internal coordinates and empirical correction by offset forces," J. Am. Chem. Soc. 114, 8191-8201 (1992).

${ }^{61}$ E. F. Koslover and D. J. Wales, "Geometry optimization for peptides and proteins: Comparison of cartesian and internal coordinates," J. Chem. Phys. 127, 234105 (2007).

${ }^{62}$ P. Maragakis, S. A. Andreev, Y. Brumer, D. R. Reichman, and E. Kaxiras, "Adaptive nudged elastic band approach for transition state calculation," J. Chem. Phys. 117, 4651-4658 (2002).

${ }^{63}$ R. Crehuet and M. J. Field, "A temperature-dependent nudged-elastic-band algorithm,” J. Chem. Phys. 118, 9563-9571 (2003).

${ }^{64}$ J.-W. Chu, B. L. Trout, and B. R. Brooks, "A super-linear minimization scheme for the nudged elastic band method," J. Chem. Phys. 119, 12708-
12717 (2003).

${ }^{65}$ B. Peters, A. Heyden, A. T. Bell, and A. Chakraborty, "A growing string method for determining transition states: Comparison to the nudged elastic band and string methods," J. Chem. Phys. 120, 7877-7886 (2004).

${ }^{66}$ E. F. Koslover and D. J. Wales, "Comparison of double-ended transition state search methods," J. Chem. Phys. 127, 134102 (2007).

${ }^{67}$ M. Griffiths, S. P. Niblett, and D. J. Wales, "Optimal alignment of structures for finite and periodic systems," J. Chem. Theory Comput. 13, 49144931 (2017).

68"OPTIM: A program for geometry optimisation and pathway calculations," http://www-wales.ch.cam.ac.uk/software.html.

${ }^{69}$ J. A. Maier, C. Martinez, K. Kasavajhala, L. Wickstrom, K. E. Hauser, and C. Simmerling, "ff14SB: Improving the accuracy of protein side chain and backbone parameters from ff99SB," J. Chem. Theory Comput. 11, 36963713 (2015).

${ }^{70}$ A. Onufriev, D. Bashford, and D. A. Case, "Modification of the generalized Born model suitable for macromolecules," J. Phys. Chem. B 104, 37123720 (2000).

${ }^{71}$ A. Onufriev, D. A. Case, and D. Bashford, "Effective Born radii in the generalized Born approximation: The importance of being perfect," J. Comput. Chem. 23, 1297-1304 (2002).

${ }^{72}$ A. Onufriev, D. Bashford, and D. A. Case, "Exploring protein native states and large-scale conformational changes with a modified generalized born model," Proteins 55, 383-394 (2004).

73“AMBER12," Case, D.A. et al.: University of California, San Francisco (2012).

${ }^{74}$ We used additional printing and debugging options to track the runs in more detail, which increases the run time significantly.

${ }^{75}$ J. M. Carr, S. A. Trygubenko, and D. J. Wales, "Finding pathways between distant local minima," J. Chem. Phys. 122, 234903 (2005).

${ }^{76}$ D. Prada-Gracia, J. Gómez-Gardenes, P. Echenique, and F. Fernando, "Exploring the free energy landscape: From dynamics to networks and back," PLoS Comput. Biol. 5, e1000415 (2009).

${ }^{77}$ D. J. Wales, "Energy landscapes: Calculating pathways and rates," Int. Rev. Phys. Chem. 25, 237-282 (2006).

${ }^{78}$ D. J. Wales, "Calculating rate constants and committor probabilities for transition networks by graph transformation (7 pages).' J. Chem. Phys. 130, 204111 (2009).

${ }^{79}$ T. D. Swinburne and D. J. Wales, "Defining, calculating, and converging observables of a kinetic transition network," Journal of Chemical Theory and Computation 16, 2661-279 (2020).

80"PATHSAMPLE: A program for generating connected stationary point databases and extracting global kinetics," http://wwwwales.ch.cam.ac.uk/software.html.

${ }^{81}$ B. Strodel, C. S. Whittleston, and D. J. Wales, "Thermodynamics and kinetics of aggregation for the gnnqqny peptide.” J. Amer. Chem. Soc. 129, 16005-16014 (2007).

${ }^{82}$ D. J. Wales, J. M. Carr, M. Khalili, V. K. de Souza, B. Strodel, and C. S. Whittleston, "Pathways and rates for structural transformations of peptides and proteins," in Proteins: Energy, Heat and Signal Flow, Computation in chemistry (CRC Press, 2009) p. 315.

${ }^{83}$ K. Röder and D. J. Wales, "Data set for "improving double-ended transition state searches for soft-matter systems",' DOI:10.5281/zenodo.3885467 (2020). 